OPEN

SUBJECT AREAS:

CANCER

AUTOPHAGY

DISEASES

CELL BIOLOGY

Received

20 July 2012

Accepted

17 October 2012

Published

13 November 2012

Correspondence and requests for materials should be addressed to M.D. (michael. detmar@pharma.ethz.

ch)

\section{The miR-290-295 cluster suppresses autophagic cell death of melanoma cells}

\author{
Yong Chen ${ }^{1}$, Ruediger Liersch² \& Michael Detmar ${ }^{1}$
}

${ }^{1}$ Institute of Pharmaceutical Sciences, Swiss Federal Institute of Technology, ETH Zurich, Zurich, Switzerland, ${ }^{2}$ Department of Medicine, Hematology and Oncology, University Hospital Muenster, Muenster, Germany.

We compared the expression levels of 307 miRNAs in six different B16F1 melanoma cell lines of differing malignant properties and found that the miR-290-295 cluster showed a strong upregulation in the more malignant $\mathrm{B} 16 \mathrm{~F} 1$ daughter cell lines. Its overexpression in $\mathrm{B} 16 \mathrm{~F} 1$ cells had no major effects on cell proliferation, migration or anchorage-independent growth, but conferred resistance to glucose starvation. This was mediated by miR-290-295-induced downregulation of several essential autophagy genes, including Atg7 and ULK1, which resulted in inhibition of autophagic cell death induced by glucose starvation. Similar effects were observed after knockdown of Atg7 or ULK1 in B16F1 melanoma cells, and after treatment with two chemical inhibitors of autophagy. Together, these results indicate that autophagy mediates cell death of melanoma cells under chronic nutrient deprivation, and they reveal an unanticipated role of the miR-290-295 cluster in conferring a survival advantage to melanoma cells by inhibiting autophagic cell death.

iRNAs are small non-coding single-stranded RNAs of 18-25 nucleotides in length that post-transcriptionally inhibit the functions of protein-coding mRNAs. Since the discovery of this class of RNA, it has become evident that miRNAs are involved in a multitude of biological processes. In particular, several miRNAs have been found to play important roles in the mediation of growth, invasion and angiogenesis of malignant tumors ${ }^{1}$ and, thus, miRNAs have become targets for developing novel anti-cancer therapeutic modalities $^{2}$.

Autophagy is a fundamental homeostatic process that is exhibited by all eukaryotic cells. In response to nutrient limitation and other stimuli, cells utilize autophagy to degrade cytoplasmic components including macromolecules and organelles to generate nutrients and energy to maintain essential activity and viability ${ }^{3,4}$. This process is orchestrated by a cohort of more than 20 autophagy-related (Atg) genes, many of which are evolutionarily conserved. Multiple protein complexes, including the ULK1/Atg1 complex and the class III PI3kinase complex in the nucleation step, and the Atg12-conjugation system and the LC3/Atg8-conjugation system in the elongation step, tightly regulate this process ${ }^{3}$.

Despite its assumed pro-survival role, increasing evidence indicates that autophagy might also act as a bona fide tumor suppressor pathway. Several tumor suppressor genes, including PTEN, TSC1, TSC2 and p53 positively regulate autophagy, while several oncogenes including Bcl-2, Bcl-XL, PI3K, AKT and mTOR are potent negative regulators ${ }^{5}$. The close overlap between regulators of autophagy and the signaling pathways that regulate tumorigenesis suggests an important involvement of autophagy in tumor pathogenesis. Genetic evidence also lends support to a potential tumor suppressive role of autophagy, as many of the known autophagy effectors and activators are located within or close to fragile sites that are associated with cancer, and are susceptible to deletions $s^{5}$. As an example, Beclin 1 (Becn1), an essential autophagy gene, is located at 17q21, a region commonly deleted in $50-70 \%$ of breast cancers and in up to $75 \%$ of ovarian cancer patients ${ }^{6}$, and mice with heterozygous disruption of Becnl showed an increased frequency of spontaneous lung cancer, hepatocellular carcinoma, and lymphoma ${ }^{7,8}$.

In the present study, we aimed to identify miRNAs that might be involved in the progression of malignant melanomas. To this end, we compared the expression levels of 307 miRNAs in six different B16F1 melanoma cell lines of differing malignant properties that were previously established in our laboratory by in vivo passaging. We found that several members of the miR-290-295 cluster showed a strong upregulation in the more malignant B16F1 daughter cell lines, when compared to the parental B16F1 line. Overexpression of miR-290-295 cluster members in B16F1 cells had no major effects on cell proliferation, migration or anchorage-independent growth in vitro, but conferred resistance to glucose starvation. This was mediated by miR-290-295-induced downregulation of several essential autophagy genes, including Atg7 and ULK1, which resulted in inhibition of autophagic cell 
A

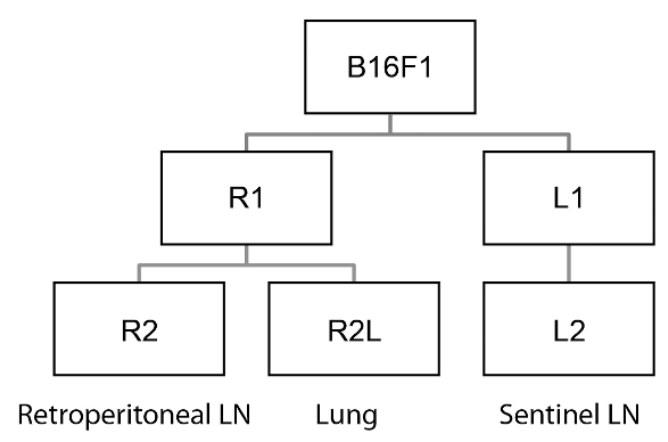

B

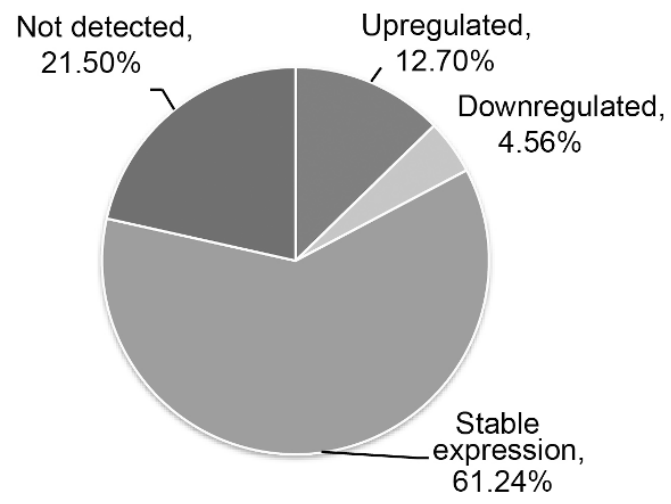

C

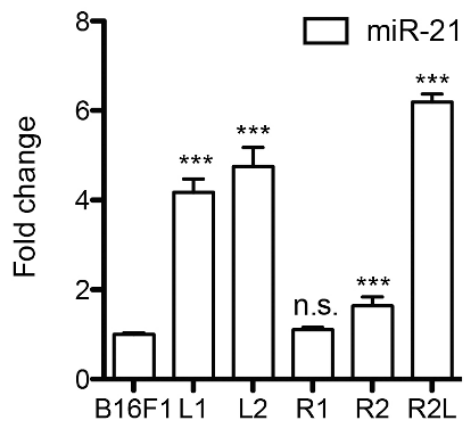

D

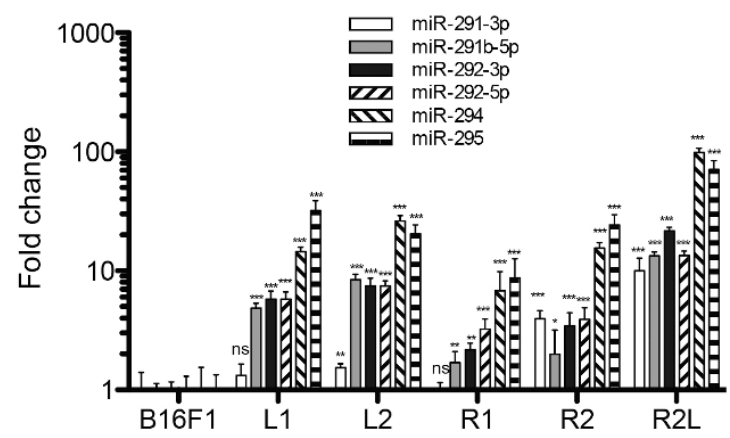

Figure 1 Quantification of 307 miRNAs with Taqman assays in six B16F1 cell lines. (A) Establishment of B16F1 daughter cell lines by in vivo passaging. (B) Pie chart shows the differential expression of miRNAs in the R2L versus the parental B16F1 cells, as determined by Taqman assay-based screening. A 2 -fold change cutoff was used. (C) Expression of miR-21 across the B16F1 lines by Taqman assays ( $\mathrm{n}=3$ ). (D) Expression of six miRNAs encoded by the miR-290-295 cluster across the B16 lines by Taqman assays $(n=3)$. Data were normalized using snoRNA135, snoRNA142, snoRNA202, snoRNA234 and snoRNA251, and results are shown as relative abundances $\pm \mathrm{SD}$. ${ }^{*} \mathrm{P} \leq 0.05 ; * * \mathrm{P} \leq 0.01 ; * * * \mathrm{P} \leq 0.001$.

death induced by glucose starvation. Inhibition of autophagic cell death was also observed after RNA interference mediated knockdown of Atg7 or ULK1 in B16F1 melanoma cells. Together, these results indicate that autophagy might mediate cell death of melanoma cells under chronic nutrient deprivation, and they reveal an important, unanticipated role of the miR-290-295 cluster in conferring a survival advantage to melanoma cells by inhibiting autophagic cell death.

\section{Results}

TaqMan array-based screening identifies differentially expressed miRNAs in melanoma lines of increased malignancy. To identify miRNAs that might regulate malignancy in melanoma, we first analyzed the global miRNA expression in B16 cell lines of differing malignant profiles that were previously established in our laboratory 9 As illustrated in Fig. 1A, the weakly metastatic B16F1 cells were initially implanted intradermally into syngeneic C57BL/6 mice, and metastases were harvested from sentinel and retroperitoneal lymph nodes to create the first generation of daughter cell lines, which were denoted as L1 and R1, respectively. After in vitro expansion, these daughter cell lines were again implanted intradermally into C57BL/6 mice. The second generation L2 line was established from a sentinel lymph node metastasis of the $\mathrm{L} 1$ line. The $\mathrm{R} 2$ line was generated from a retroperitoneal lymph node metastasis of R1, and the R2L line was from a lung metastasis of R1. These daughter cell lines have a more metastatic behavior in vivo than the parental B16F1 cells and express increasing levels of SPP1/osteopontin ${ }^{9}$ (Supplementary Fig. 1A), which is associated with metastasis in a wide range of solid tumors $^{10}$. The lowest level of osteopontin expression was found in parental B16F1 cells, and the highest level in R2L cells. Whereas there were no major differences between the cell lines in cell proliferation in 2-D culture ( ${ }^{9}$ and data not shown), R2 cells and, more strikingly, R2L cells formed large colonies in growth factor-reduced Matrigel in 3D culture, whereas parental B16F1 cells did not efficiently form colonies (Supplementary Fig. 1B). Together, these results indicate that the $\mathrm{B} 16$ cells lines, that were established by in vivo selection and consecutive in vitro culture, exhibit different degrees of malignancy.

We next investigated the miRNA expression levels in all six B16F1 lines, using a panel of TaqMan assays for 307 mouse miRNAs. A twofold change cutoff was chosen for classification of candidate miRNAs as downregulated or upregulated (less than $50 \%$ or more than $200 \%$ expression compared to parental B16F1 cells). We found that $61.2 \%$ of the miRNAs were stably expressed and $21.5 \%$ were not detected in B16F1 or R2L cells; $4.6 \%$ were downregulated and $10.4 \%$ were upregulated in R2L cells compared to B16F1 cells (Fig. 1B). Among the miRNAs differentially expressed (Supplementary Fig. 1C), miR-21 and the miR-290-295 cluster were of particular interest. Expression of miR-21, a well-known oncomiR that is overexpressed in most solid tumors analyzed ${ }^{11}$, was significantly higher in 4 out of 5 daughter lines than in the parental B16F1 cells (Fig. 1C). The expression of the miR-290-295 cluster, which encodes a family of miRNAs that are expressed de novo during early embryogenesis and have been described as specific for mouse embryonic stem cells and embryonic carcinoma cells ${ }^{12}$, was strongly upregulated in all daughter cell lines, with the highest expression in R2L cells (Fig. 1D). In particular, miRNA-294 and miR-295 showed the highest upregulation.

The miR-290-295 cluster renders melanoma cells resistant to glucose deficiency. The miRNA-290-295 cluster genes were next 
A
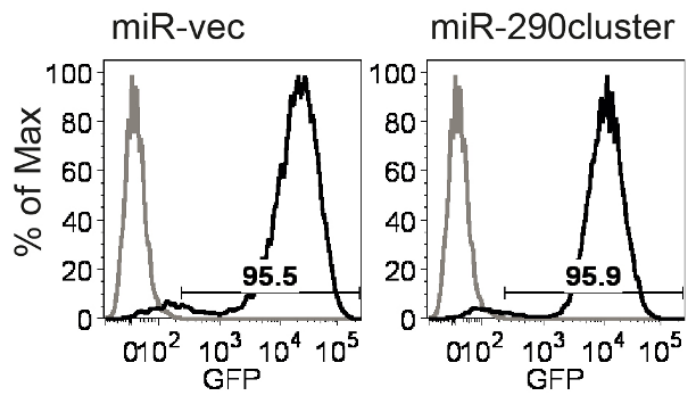

C

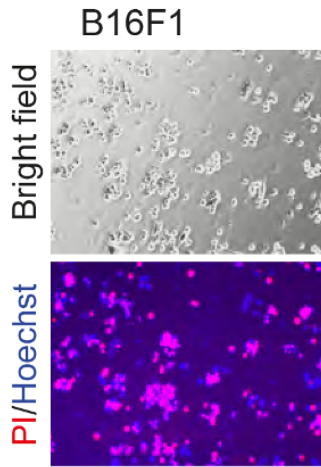

R2L

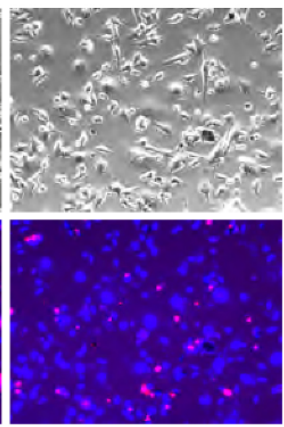

E

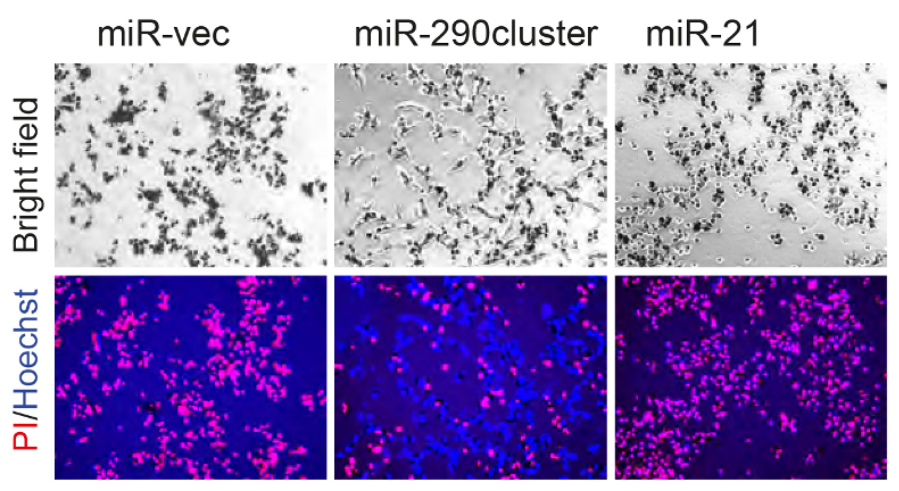

B
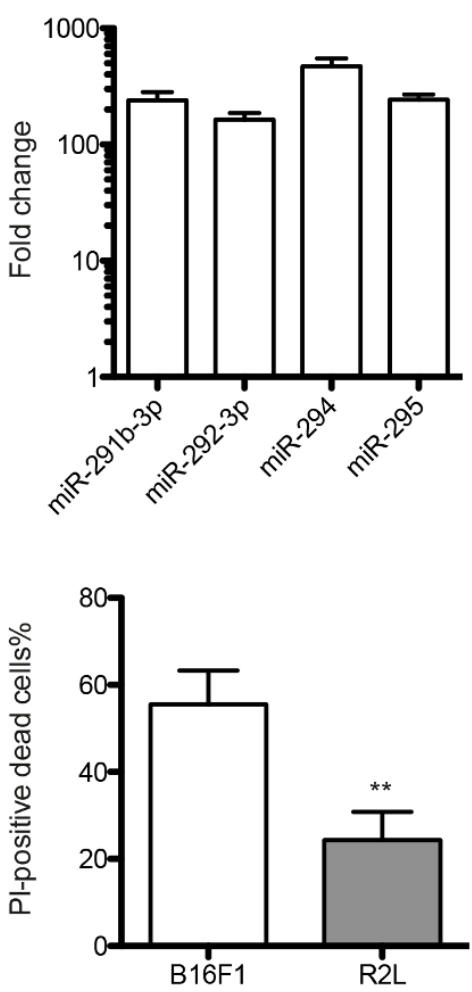

$\mathrm{F}$

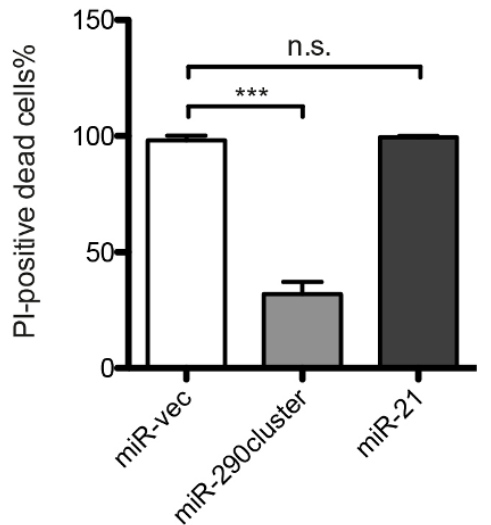

Figure $2 \mid$ The miR-290-295 cluster confers resistance to glucose starvation to B16F1 cells. (A) B16F1 cells were infected with a retrovirus encoding the miR-290-295 cluster or control miR-vec retrovirus, both of which also encode GFP. Three days after infection, efficiency of infection was monitored by tracking GFP expression using flow cytometry. The gray line represents non-infected cells, whereas the black line represents cells infected by the miRNA retrovirus. Percentage of GFP-positive cells is indicated. (B) Fold change of four miR-290-295 cluster miRNAs in B16F1 cells three days after infection $(n=3)$. (C and D) R2L cells showed a survival advantage over B16F1 cells under glucose starvation. After two days of starvation, cells were stained with Hoechst 33342 and PI $(n=3)$. Representative pictures were taken $(\mathrm{C})$ and ratios of PI-positive dead cells over total cells were calculated after counting (D). (E and F) B16F1 cells infected with miR-vec, the miR-290-295 cluster or miR-21 retrovirus were glucose starved for three days and stained with Hoechst 33342 and PI $(n=3)$. Representative images are shown $(E)$ and the percentage of PI-positive dead cells was calculated (F). Data are shown as means $\pm \mathrm{SD} . * * \mathrm{P} \leq 0.01 ; * * * \mathrm{P} \leq 0.001$.

cloned into a retroviral construct, MDH1-PGK-GFP 2.0 that also encodes GFP, and virus was produced to overexpress the miRNAs in B16F1 cells, achieving a high infection efficiency ( 96\%) (Fig. 2A) and a strong overexpression (Fig. 2B). Overexpression of the miR290-295 cluster did not promote the growth of B16F1 cells in Matrigel or the anchorage-independent growth in soft agar (Supplementary Fig. 2A). Accordingly, parental B16F1 and R2L cells did not show significant differences in transwell cell migration or invasion through the Matrigel (Supplementary Fig. 2B, C), and overexpression of the
miRNA-290-295 cluster genes also did not affect transwell cell migration or invasion (Supplementary Fig. 2D, E).

We next investigated the capability of parental B16F1 cells and of R2L cells to survive under glucose deprivation. We found that after three days of culture in glucose-deficient growth medium, the more malignant R2L cells showed a significantly stronger capability to survive than B16F1 cells (Fig. 2C, D). Importantly, B16F1 cells overexpressing the miR-290-295 cluster were significantly more resistant to glucose deprivation than control infected B16F1 cells 
A

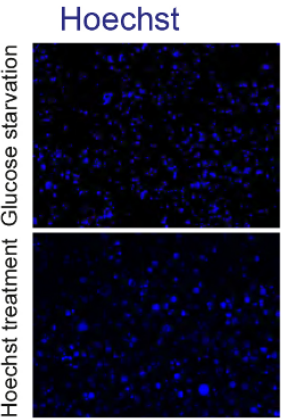

PI

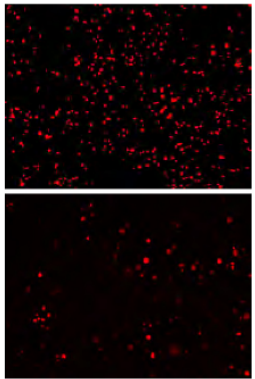

C

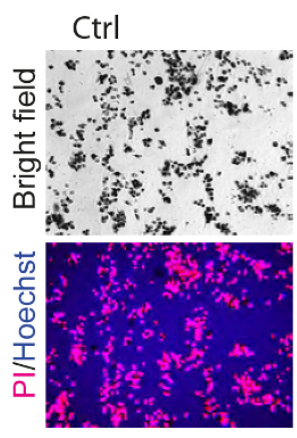

3-MA

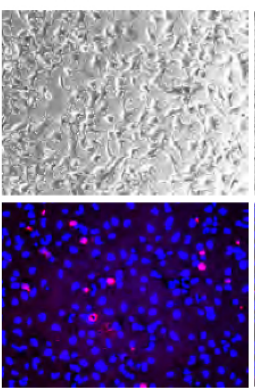

LY294002

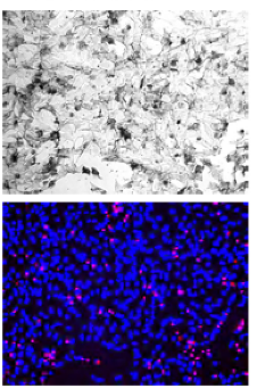

B

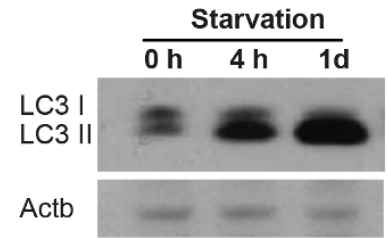

D

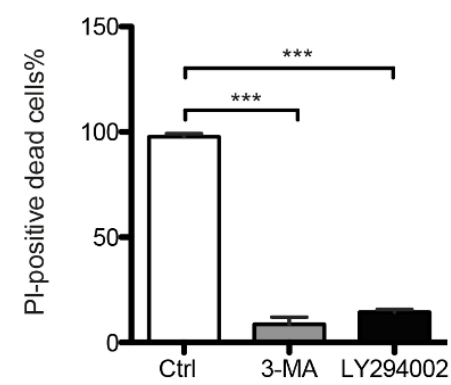

G

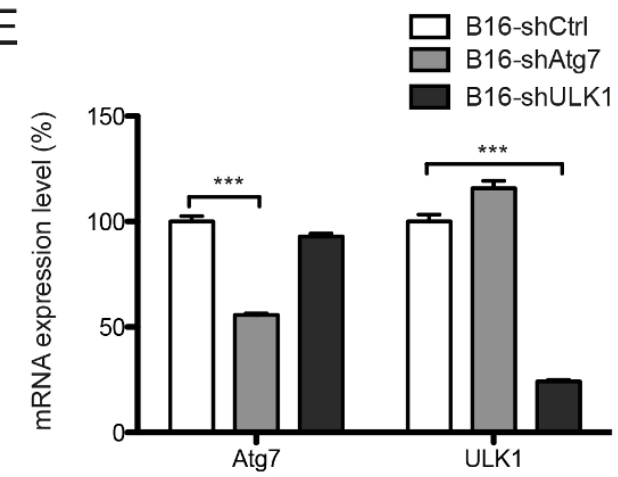

F
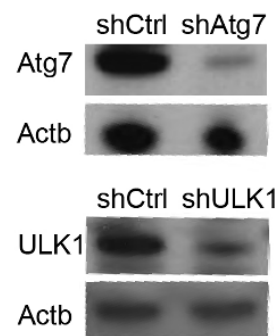

LC3II

Actb

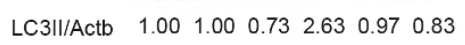

$\mathrm{H}$

shCtrl

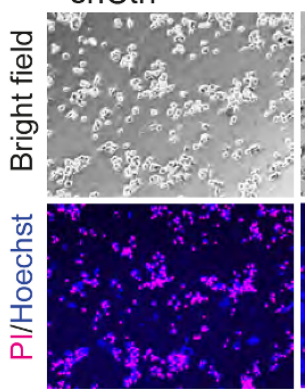

shAtg7

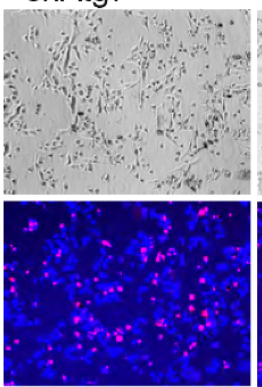

shULK1

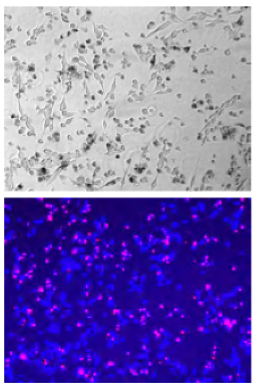

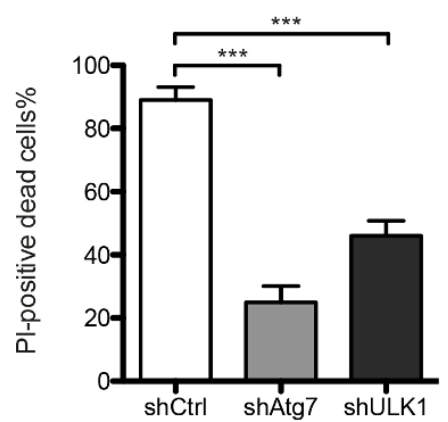

Figure 3 Glucose starvation induces autophagic cell death in B16 cells. (A) B16F1 cells did not show nuclear condensation typical of apoptosis after glucose starvation. Cells were glucose starved for three days and stained with Hoechst 33342 and PI. The PI-positive dead cells showed weak nuclear staining by Hoechst 33342 (upper panels), whereas apoptotic cells induced by Hoechst 33342 treatment $(1 \mu \mathrm{g} / \mathrm{ml}, 20 \mathrm{~h}$ ) showed nuclear condensation (lower panels). (B) Glucose starvation induced strong autophagy, evidenced by an increased amount of LC3-II that correlates with autophagic activity. Whole cell lysates of B16F1 cells (non-starved or glucose starved for $4 \mathrm{~h}$ or $24 \mathrm{~h}$ ) were subjected to Western blot using an antibody recognizing both LC3-I and LC3-II. (C and D) 3-MA and LY294002, two autophagy inhibitors, significantly rescued glucose starvation-induced cell death. Cells were glucose starved in the presence of 3-MA $(10 \mathrm{mM})$ or LY294002 $(10 \mu \mathrm{M})$ for three days, and stained with Hoechst 33342 and PI $(\mathrm{n}=3)$. Representative images were taken (C) and the percentage of PI-positive dead cells was calculated (D). (E, F, G) Efficient inhibition of autophagy by knockdown of the autophagy genes Atg7 and ULK1 with lentivirus-mediated shRNA. B16 cells were infected with shRNA lentivirus and selected with puromycin $(2 \mu \mathrm{g} / \mathrm{ml})$ for $10 \mathrm{~d}$, followed by qPCR (E) and Western blot (F) confirmation of knockdown. The cells were glucose starved for 24 hours and subjected to LC3 immunoblot (G). (H and I) Atg7 and ULK1 knockdown significantly promoted cell survival after three days of glucose starvation $(\mathrm{n}=3)$. Representative images $(\mathrm{H})$ and percentage of PI-positive dead cells (I) are shown. Data are shown as means \pm SD. ${ }^{* * *} \mathrm{P} \leq 0.001$. 
(Fig. 2E, F). After three days of glucose starvation, many of the cells infected with the miR-vec control virus became detached from the plate and underwent cell death as revealed by the strong PI staining. In contrast, most of the B16F1 cells overexpressing the miR-290-295 cluster still remained adherent and viable (Fig. 2E, F). In contrast, miR-21, which was also upregulated in R2L cells compared to B16F1 cells, did not show such an effect (Fig. 2E, F).

B16F1 cells undergo autophagic cell death in response to chronic glucose deprivation. To further study the potential mechanisms underlying the rescuing effects of the miR-290-295 cluster on the response of melanoma cells to glucose starvation, we characterized the effects induced by glucose deprivation in more detail. When cultured in glucose-free medium for three days, massive cell death of B16F1 cells was evident by the positive PI staining (Fig. 3A upper panels). Interestingly, the dead (PI-positive) cells were stained only faintly by Hoechst 33342, in contrast to apoptotic cells that typically show nuclear condensation ${ }^{13,14}$. As a control for apoptosis-induced changes, we also treated B16F1 cells with Hoechst 33342, an inhibitor of topoisomerase I that induces apoptosis in cancer cells ${ }^{15}$. As shown in Fig. 3A (lower panels), nuclear condensation was apparent in Hoechst 33342-treated cells. These findings indicate that glucose starvation induced a non-apoptotic cell death pathway.

Glucose starvation has been reported to induce autophagy, and in some cases non-apoptotic autophagic cell death ${ }^{16,17}$. This prompted us to investigate whether autophagy might mediate glucose starvation-induced cell death in B16F1 cells. We first addressed whether autophagy was induced in response to glucose starvation by studying the processing of the microtubule-associated protein 1 light chain 3 (LC3). Two forms of LC3, called LC3-I and LC3-II, are produced post-translationally. LC3-I is cytosolic and is lipidated and converted to LC3-II that is associated with autophagosome membranes when autophagy occurs ${ }^{18}$. Thus, the amount of LC3-II correlates with autophagic activity and has therefore been widely used to measure autophagic activity ${ }^{18,19}$. We found that glucose starvation for four hours resulted in a strong accumulation of LC3-II (Fig. 3B), indicating a potent autophagic activity. Prolonged starvation for one day led to a dramatic further increase in autophagy (Fig. 3B).

We next inhibited autophagy with LY294002 $(10 \mu \mathrm{M})$ or 3-methyladenine (3-MA; $10 \mathrm{mM})$, both of which are widely used as autophagy inhibitors ${ }^{20,21}$. As shown in Fig. 3C, both compounds significantly rescued B16F1 cells from cell death induced by glucose deprivation. Consistent with this, LY294002 and 3-MA also significantly increased the clonogenicity of B16F1 cells after glucose starvation (Supplementary Fig. 3A, B), indicating that autophagy is responsible for the cell death after prolonged glucose starvation. We then knocked down two genes essential for autophagy, namely Atg7 and ULK1, by lentivirus-mediated shRNA transduction. B16F1 cells were transduced and selected with puromycin to enrich the infected pools. The cells infected with the shCtrl virus maintained a comparable sensitivity to glucose starvation as the non-infected cells (Supplementary Fig. 3C, D). After successful knockdown of Atg7 or ULK1 (Fig. 3E, F), induction of autophagy was reduced in response to glucose starvation (Fig. 3G), and the cells survived significantly better than the control cells (Fig. 3H, I). They also showed a significantly higher clonogenicity after glucose starvation (Supplementary Fig. 3E, F). Together, these data reveal that B16F1 cells undergo autophagic cell death in chronic glucose deprivation.

The miR-290-295 cluster inhibits autophagy by repressing multiple autophagy-related genes. To explore whether and how the miR290-295 cluster might regulate the autophagic process in B16F1 cells, we next investigated whether these miRNAs were predicted to target autophagy-related genes. For comparison, miR-21 and miR-211, that are oncogenic ${ }^{22}$ or tumor suppressive ${ }^{23}$ in melanoma, respectively, were also included in the analysis. Using TargetScanMouse Release $6.0^{24}$, we analyzed whether there is a complementarity between the miRNA seed
Table 1 | Prediction of miRNA binding sites in the 3'UTRs of key autophagy-related genes' by TargetScanMouse Release 6.0

$\begin{array}{llll}\text { Gene miR-290-295 miR-21 miR-211 } & \end{array}$

I. Nucleation Step:

ULK/Atg I complex:

Atg 1 /ULK 1

$\operatorname{Atg} 13$

Atg 101

FIP200/Rblcc l

Class III

AMBRA
Atg 14

Becn 1/Atg6

p150

Vps34

Others:

Atg $2 b$

Atg9a

DFCP 1

VMP1

WIPI2

II. Elongation Step:

Atg 12 -conjugation

system:

Atg5

Atg7

Atg 10

Atg 12

Atg $16 \mathrm{LI}$

LC3/

Atg $4 a$

Atg $4 b$

Atg $4 \mathrm{c}$

Atg $4 d$

Atg7

LC3/Atg 8

Yes $/(1 \mathrm{C})^{2,3}$
No /
No / 0
Yes / $(3 A+1 C)$
mplex:

No / 0

No $/ 0$

No $/ 0$

No / 0

No / 0

$\begin{array}{ll}\text { Yes } /(1 \mathrm{~A}+1 \mathrm{C}) & \text { No } / 0 \\ \text { Yes } /(1 \mathrm{~B}+4 \mathrm{C}) & \text { No } / 0\end{array}$

$\mathrm{No} / \mathrm{O}$

No / $O$

No / O

No / O

Yes / (2A)

Yes / (1A)

No / O

Yes / (1A)

No / O

No / 0

Yes / (1A)

No / O

No / O

No $/ 0$

No / O

No / O

No / O

$\mathrm{No} / \mathrm{O}$

No $/ 0$

No $/ 0$

No $/ 0$

No / O

No $/ 0$

No $/ 0$

No $/ 0$

Yes / (1B)

No $/ 0$

No / O

'The gene list is adopted from Reference 19.

${ }^{2}$ Gene is predicted or not to be targeted by miRNA / (number and types of predicted binding sites) ${ }^{3}$ Classification of binding sites: A: predicted only in mouse; B: in mouse and rat; C: in mammals.

and the 3'UTR, and the conservation of putative binding sites across different species (Table 1). Since binding sites that are conserved across different species are more likely to be biologically functional, as they may have been maintained for important functions under selective pressure during evolution ${ }^{25}$, we classified the putative binding sites into three categories: predicted only in mouse (denoted as class A), conserved only in mouse and rat (denoted as class B) and conserved in mammals (denoted as class C). The miR-290-295 cluster was predicted to target 17 out of the 25 autophagy-related genes essential for forming the ULK1/Atg1 complex, the class III PI3-kinase complex, the Atg12conjugation system, and the LC3/Atg8-conjugation system, which are all key to autophagy ${ }^{19}$. In contrast, miR-21 and miR-211 were only predicted to target 2 or 1 autophagy-related genes, respectively (Table 1). This striking enrichment of autophagy-related genes in the predictions strongly indicated a role of the miR-290-295 cluster in regulating autophagy.

To study the potential regulation of these genes by miRNAs, we next cloned the 3'UTRs of mouse Atg4B, Atg5, Atg7, Atg12, Atg16L1 and Becn 1 into the psiCHECK-2 dual-luciferase reporter, to generate artificial 3'UTRs of Renilla luciferase. The psiCHECK-3'UTR reporters were then transfected into B16F1 and R2L cells. The relative activity of Renilla luciferase was normalized to that of the empty psiCHECK-2 vector, in which Renilla luciferase does not contain a 3'UTR and is not regulated by any miRNA. This ratio indicates whether and to which extent these genes are regulated by the endogenous miRNAs in cells. The activities of most psiCHECK-3'UTR 
reporters except Atg12 were significantly more strongly suppressed in R2L cells compared to B16F1 cells, suggesting that they were regulated by miRNAs differentially expressed between these two cell lines (Fig. 4A).

To study whether the miR-290-295 cluster might indeed regulate these essential autophagy genes, we co-transfected individual psiCHECK-3'UTR reporters (Atg4b, Atg5, Atg7, Becn1 and ULK1) and plasmids encoding the miRNAs predicted to target these genes into B16F1 cells, and performed dual-luciferase assays. As shown in Fig. 4B, all autophagy-related genes tested in this assay, namely Atg4b, Atg5, Atg7, Becn1 and ULK1 were significantly downregulated by the miR-290-295 cluster.

The regulation of these target genes was further studied at the protein level by Western blot analyses. B16F1 cells were infected with miR-vec or the miR-290-295 cluster retrovirus. MiR-142, which encodes miR-142-3p and miR-142-5p, the two most upregulated miRNAs in R2L cells compared to B16F1 cells (Supplemental Fig. 1C), was also included. Among the proteins examined (Atg5, Atg7, Atg16L1, Becn1 and ULK1), Atg7 and ULK1 were strongly reduced by the miR-290-295 cluster, consistent with the dual-luciferase assay results. A reduction of Atg5, Atg16L1 or Becn1, however, was not observed at the Western blot level. Interestingly, Atg7 was also reduced by miR-142 (Fig. 4C).

We next investigated whether these miRNAs might regulate autophagic activity. To this end, we treated B16F1 cells - infected with the miR-vec, miR-142 or the miR-290-295 cluster retrovirus with $0.5 \mu \mathrm{g} / \mathrm{ml}$ of rapamycin for 24 hours, and determined LC3 conversion by Western blot. Rapamycin inhibits $\mathrm{mTOR}^{26}$ and is known to induce autophagy in B16 cells ${ }^{27}$. We found that the LC3II amount was greatly upregulated after rapamycin treatment, indicating enhanced autophagic activity (Fig. 4D). The cells overexpressing the miR-290-295 cluster had less LC3-II, indicating reduced autophagic activity in response to rapamycin treatment, while cells overexpressing miR-142 did not show this effect (Fig. 4D). Together, these findings establish a role of miR-290-295 in repressing rapamycin-induced autophagy.

We next incubated B16F1 cells that were infected with either miRvec, the miR-290-295 cluster or miR-21 retrovirus, in glucose-free medium for 24 hours and studied their autophagic activity. As shown in Fig. 4E, overexpression of the miR-290-295 cluster strongly inhibited autophagy, as evidenced by the reduced LC3-II amount compared to control cells. In contrast, miR-21, which did not rescue glucose starved cells, did not regulate autophagy. We also performed an autophagy flux assay using the lysosomal protease inhibitors E64d and pepstatin $\mathrm{A}^{19}$. As shown in Fig. 4F, the miR-290-295 cluster reduced autophagy induction by glucose starvation, and treatment with E64d and pepstatin A slightly increased this effect.

Together, these findings indicate that the miR-290-295 cluster regulates multiple autophagy genes and is able to suppress autophagy induced by rapamycin stimulation and glucose starvation.

\section{Discussion}

In this study, by using a TaqMan-based qRT-PCR profiling platform to quantify the expression levels of 307 miRNAs in parental B16F1 mouse melanoma cells and in five more daughter cell lines of increasing malignancy that were established through in vivo selection ${ }^{9}$, we found that the miR-290-295 cluster was markedly upregulated in the more malignant melanoma cell lines. Previously, the miRNAs of the miR-290-295 cluster have been characterized as mouse embryonic stem cell-specific miRNAs ${ }^{12}$. They represent the most abundant miRNAs, constituting more than $70 \%$ of the entire miRNA population in mouse embryonic stem cells, and they have been found to be downregulated during differentiation ${ }^{12}$. In mouse embryonic stem cells, the miR-290-295 cluster has been described to promote rapid proliferation $^{28}$ and cell survival during genotoxic stress ${ }^{29}$. In human germ cell tumors, the human orthologs of the miR-290-295 cluster, namely the miR-371-373 and miR-302 clusters, have been found to be overexpressed when compared to normal tissues, and they have been reported to coordinately downregulate mRNAs that are involved in multiple biologically significant pathways ${ }^{30}$. MiR-372 and miR-373 were found to inhibit the p53 pathway in germ cell tumors $^{31}$ but, to our knowledge, they have not been directly implicated in the pathogenesis of non-germline solid tumors.

The results of our study establish, for the first time, a link between the miR-290-295 cluster and autophagy. Autophagy is being increasingly implicated in mediating either cell survival or cell death. Its most fundamental and conserved function in cancer cells is likely to respond to and to buffer metabolic stress. Inhibition of autophagy has been previously linked to increased apoptosis in some types of cancer cells. For instance, colorectal cancer cells activate autophagy in response to glucose starvation, and inhibition of autophagy with 3MA or RNA interference mediated knockdown of Atg7 induced massive apoptosis ${ }^{32}$. In agreement with these reports, we also found that in HeLa E1 cervical carcinoma cells under persistent glucose starvation, dead cells presented typical characteristics of apoptosis, such as nuclear condensation and fragmentation, and that inhibition of autophagy with chemical inhibitors (3-MA and LY294002) sensitized cells to death (Supplementary Fig. 4). However, autophagy has also been found to act as a bona fide cell death mediator in several cell types. For instance, treatment of mouse L929 fibroblastic cells with the pan-caspase inhibitor $\mathrm{z}-\mathrm{VAD}$-fmk causes autophagic cell death, which can be rescued by RNA interference mediated knockdown of the autophagy genes Becn 1 or Atg $7^{33}$. Similarly, rat cardiomyocytederived H9c2 cells also undergo autophagic cell death under glucose deprivation ${ }^{16}$, and low intracellular glucose levels may also lead to autophagic cell death in some cancer cells ${ }^{34}$.

The results of our study reveal that B16F1 melanoma cells undergo autophagic cell death in response to chronic starvation, which could be rescued by both pharmacological (3-MA and LY294002) and genetic (shAtg7 and shULK1) inhibition of autophagy. Furthermore, we found, for the first time, that the miR-290-295 cluster confers cellular resistance to glucose deprivation (Fig. 5). We found that this cluster is predicted by TargetscanMouse Release 6.0 to target the majority of the essential genes for autophagy, including Atg2b, Atg4a, Atg4b, Atg4c, Atg4d, Atg5, Atg7, Atg9a, Atg10, Atg12, Atg14, Atg16L1, Becn1, FIP200, ULK1, VMP1 and WIPI2, in contrast to the oncogenic miR-21 which is predicted to only target DFCP1 and LC3. Consistent with this prediction, we found that overexpression of miR-21 did not promote B16F1 cell survival under glucose deprivation. It is of interest that the expression of several autophagy genes was reduced in the highly malignant R2L daughter cell line compared to parental B16F1 cells, as indicated by the results of dual-luciferase reporter assays. Importantly, overexpression of miRNAs of the miR290-295 cluster in B16F1 cells resulted in suppression of multiple autophagy genes, as seen by both dual-luciferase assays and Western blot analyses. Consequently, overexpression of miRNAs of the miR290-295 cluster potently inhibited autophagy induced by rapamycin treatment and by glucose starvation. These findings represent an example that miRNAs can simulataneously fine tune multiple genes in a distinct pathway and thereby exert significant cellular functions.

It has been reported that induction of autophagy during extracellular matrix detachment promotes cell survival, and that knockdown of distinct autophagy genes reduced the clonogenic survival of epithelial cells and fibroblasts ${ }^{35}$. However, we found that overexpression of the miR-290-295 cluster did not affect their survival and colony formation in soft agar. These data suggest that while endorsing resistance to glucose deprivation, these miRNAs do not compromise $\mathrm{B} 16 \mathrm{~F} 1$ cell survival in other conditions.

Tumor cells suffer from metabolic stress when growth progresses towards a mass in the range of $1 \mathrm{~mm}$ in diameter before efficient blood supply is established by angiogenesis ${ }^{36}$. Even after successful induction of tumor angiogenesis, tumor blood vessels 
A

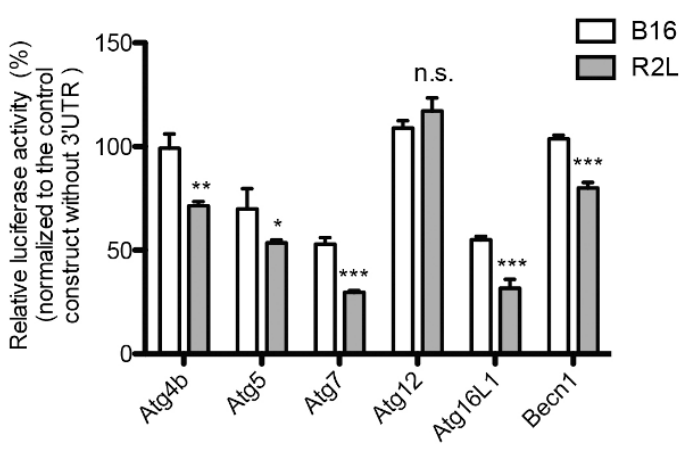

C

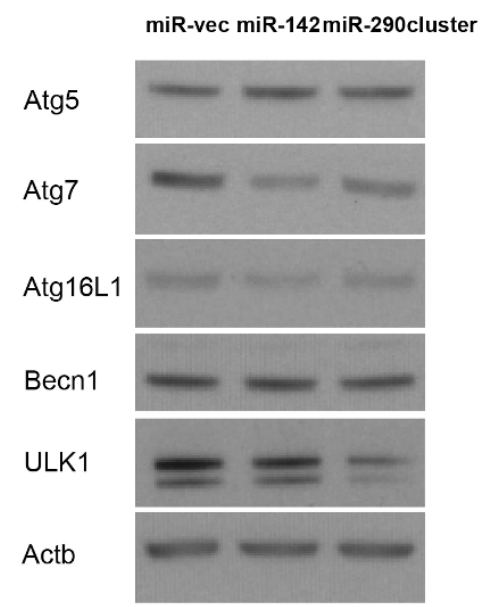

B

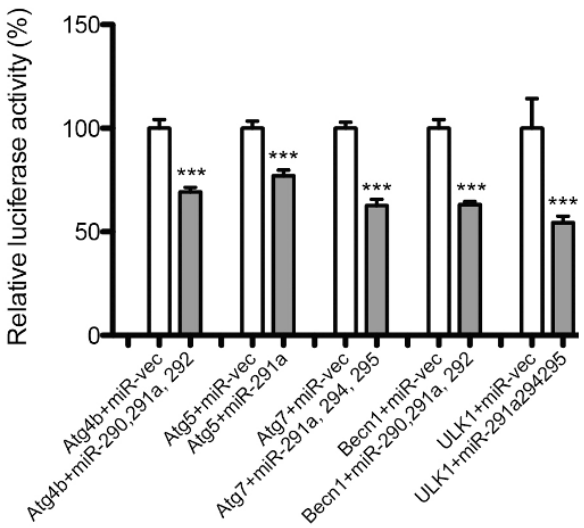

D

Rapamycin: $\frac{\text { miR-vec }}{-}+\frac{\text { miR-142 }}{-}+\frac{\text { miR-290cluster }}{-}+\frac{+}{-}$

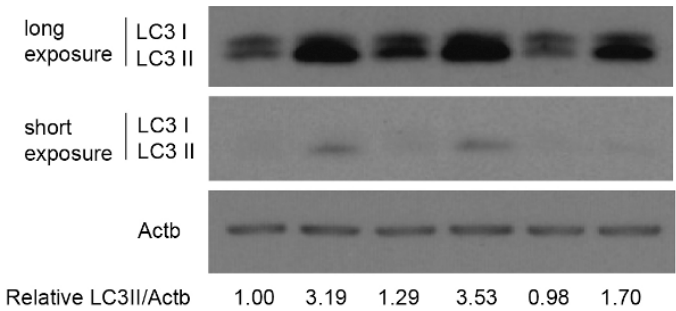

E
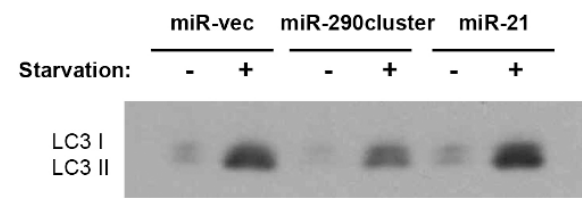

Actb

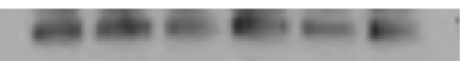

Relative LC3II/Actb $\quad 1.00 \quad 3.01 \quad 0.85 \quad 1.40 \quad 1.40 \quad 3.60$

F

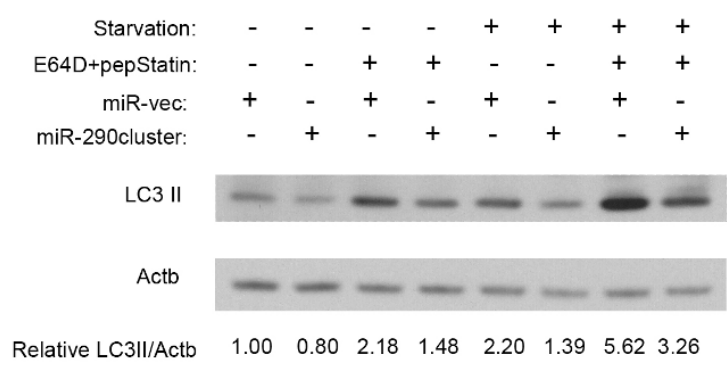

Figure $4 \mid$ The miR-290-295 cluster inhibits autophagy. (A) 3'UTRs of autophagy related genes were repressed in R2L cells as compared to B16 cells. 3'UTR luciferase reporters were transfected into cells and dual-luciferase assays were carried out $(n=3)$. For each 3'UTR, renilla luciferase values were first normalized to firefly luciferase activity, and the calculated relative luciferase activity of each 3'UTR reporter was further normalized to that of the control reporter without any 3'UTR. (B) The miR-290-295 cluster repressed autophagy genes in the dual-luciferase assays. The 3'UTR luciferase reporters were co-transfected with miRNA plasmids that were predicted to target certain genes, and luciferase activities were assayed $36 \mathrm{~h}$ post transfection $(\mathrm{n}=3$ ). For all luciferase activity assays, renilla luciferase values were normalized to firefly luciferase activity and plotted as relative luciferase activity.

(C) The miR-290-295 cluster suppressed expression of Atg7 and ULK1. Whole cell lysates of B16F1 cells infected with the indicated miRNA retrovirus were subjected to Western blots. (D, E, F) The miR-290-295 cluster suppressed rapamycin or glucose starvation-induced autophagy. Infected B16F1 cells were treated with rapamycin $(0.5 \mu \mathrm{g} / \mathrm{ml})$ for $24 \mathrm{~h}(\mathrm{D})$ or were glucose starved for $24 \mathrm{~h}$ without $(\mathrm{E})$ or with lysosomal protease inhibitors E64d (10 $\mu \mathrm{g} / \mathrm{ml})$ and pepstatin A $(10 \mu \mathrm{g} / \mathrm{ml})(\mathrm{F})$, and subjected to Western blot using an antibody recognizing both LC3-I and LC3-II. Actb was used as a loading control. Data are shown as means $\pm \mathrm{SD} .{ }^{*} \mathrm{P} \leq 0.05 ; * * \mathrm{P} \leq 0.01 ; * * * \mathrm{P} \leq 0.001$. 

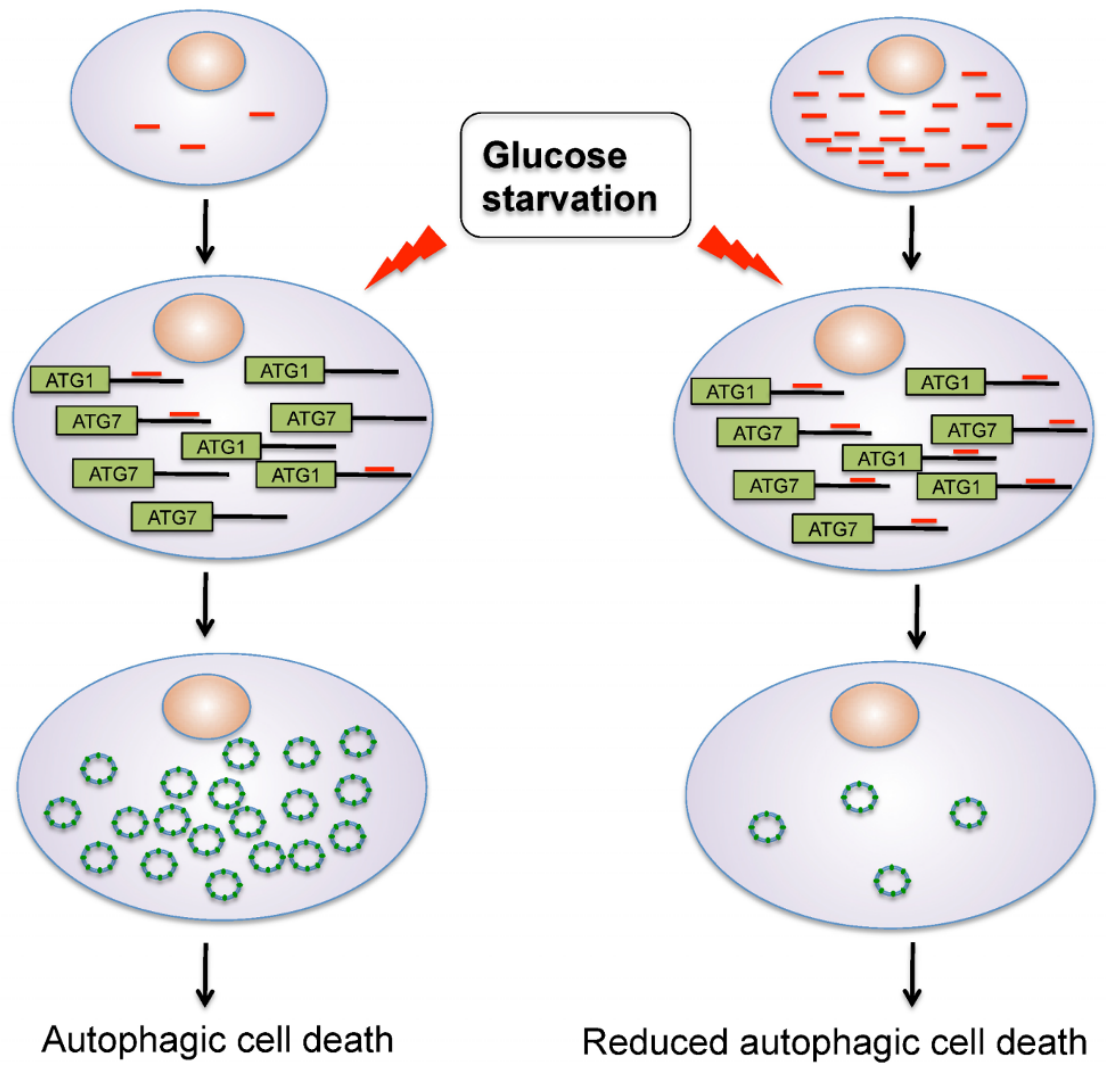

Reduced autophagic cell death

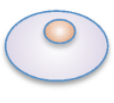

B16F1 melanoma cell

Autophagy gene with its 3' UTR

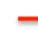

miRNA of the miR-290-295 cluster

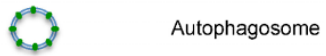

Figure 5 Schematic representation of the role of the miR-290-290 cluster in the inhibition of autophagic cell death. B16F1 cells undergo autophagic cell death under chronic glucose starvation. The miR-290-295 cluster reduced the autophagic activity by simultaneously repressing multiple autophagy genes and thus confers resistance to glucose starvation to B16F1 cells.

are largely abnormal, which results in metabolic stress in established tumors ${ }^{37}$. Moreover, the preference for glycolysis (which is $\sim 18$-fold less efficient at ATP production relative to mitochondrial oxidative phosphorylation) and the unregulated tumor cell growth also contribute to the high metabolic demands and the consequent metabolic stress of tumor cells ${ }^{38}$. Thus, the capability of the miR-290-295 cluster to enable B16F1 cells to survive glucose starvation might also endow these cells with a significant advantage for survival and progression in vivo.

A number of mechanisms have been proposed to explain tumor suppressive functions of autophagy in several types of cancer. Our results reveal that at least in $\mathrm{B} 16 \mathrm{~F} 1$ melanoma cells, prolonged metabolic stress causes autophagic cell death. The miR-290-295 cluster, which is strongly upregulated in the more malignant R2L daughter cells, helps cells buffer glucose limitation by simultaneously targeting multiple autophagy genes and therefore restraining autophagic cell death. Although the roles of these miRNAs and autophagy still remain to be further investigated in vivo and in other melanoma cell lines, our work adds a new dimension to the understanding of the roles of autophagy and miRNAs in tumor progression.

\section{Methods}

Cells. The parental B16F1 cells were kindly gifted by Dr. Ralph Weissleder (Harvard Medical School, Boston). The daughter cell lines (L1, L2, R1, R2 and R2L) were established in our laboratory as recently reported ${ }^{9}$. Cells were cultured in Dulbecco's modified Eagle medium (DMEM; Gibco, UK) supplemented with $10 \%$ fetal bovine serum (FBS; Gibco).

RNA and protein isolation. Total cellular RNA was isolated from 100-mm tissue culture dishes at 80 to $90 \%$ confluency with the mirVana PARIS Kit (Ambion, USA). The quality and concentration of RNA was measured using a NanoDrop ND-1000 spectrophotometer (Witec AG, Switzerland). Only RNA samples of high quality were used. Total protein was extracted using the Cell Disruption Buffer (mirVana PARIS Kit) supplemented with 1x Complete Protease Inhibitor Cocktail \& PhosSTOP Phosphatase Inhibitor Cocktail (Roche, Switzerland). The protein concentration was determined with the Pierce BCA Protein Assay Kit (Pierce, USA).

TaqMan miRNA assays. Quantification of miRNAs was done in triplicates using two-step TaqMan MicroRNA Assays (Applied Biosystems, part number 4383444). Reverse transcription reactions were performed using $10 \mathrm{ng}$ of total RNA and the microRNA Reverse Transcription kit (Applied Biosystems). MiRNA expression levels of technical triplicates were determined using a 7900HT Fast Real-Time PCR System (Applied Biosystems), and $\mathrm{C}_{\mathrm{T}}$ values were acquired after 40 cycles using SDS 2.2 software (Applied Biosystems). Five small nucleolar RNAs, namely snoRNA135, snoRNA142, snoRNA202, snoRNA234 and snoRNA251 were used as loading controls. For analysis, SDS 2.2 software was used, and when a miRNA $\mathrm{C}_{\mathrm{T}}$ value was undetermined in all technical replicates, a $C_{T}$ value of 41 was assigned. The $2^{-\Delta \Delta C T}$ 
method was used for quantification. Heatmap was visualized with the GenePattern software $^{39}$ using the HeatMapViewer module.

Quantitative real-time PCR of mRNAs. Total RNA was isolated using the RNeasy Mini Kit (QIAGEN), reverse transcribed into cDNA using the High-Capacity cDNA Reverse Transcription Kit (Applied Biosystems), and Sybr Green qPCR assays were performed in triplicates using an Applied Biosystems 7900HT Fast Real-Time PCR System. Qiagen QuantiTect Primer Assays were used. For all RT-PCR analyses, ACTB and 36B4 mRNAs were used for normalization.

Cloning of miRNAs and 3'UTRs. For cloning of miRNAs and 3'UTRs, genomic DNA was extracted from B16F1 mouse melanoma cells with the DNeasy Kit (Qiagen) and was used as template. MDH1-miR-142-PGK-GFP was purchased from Addgene (Plasmid \#11377). Other miRNAs were cloned into the XhoI \& EcoRI sites of the retroviral plasmid MDH-PGK-GFP_2.0 (Addgene Plasmid \#11375). 3'UTRs were cloned into psiCHECK-2 (Promega) behind the stop codon of Renilla luciferase. Plasmids were further verified by gel electrophoresis after double digestion and by sequencing (Synergene, Switzerland). The PCR primers used are listed in Supplementary Table S1.

Virus production and infection. To produce miRNA-overexpressing retroviruses and shRNA-overexpressing lentiviruses, transfer vectors and proper packaging plasmids (for retrovirus production: pVsvg and pMLV; for lentivirus production: psPax2 and pMD2.G) were co-transfected into HEK293T cells with polyethylenimine (Polysciences, USA). Supernatant containing virus particles was collected, aliquoted and used for infection. Cells were infected for 6-8 hours with viral supernatant: fresh medium $=1: 1$ with $8 \mu \mathrm{g} / \mathrm{ml}$ of polybrene (Sigma), and used for assays at least three days after infection to allow efficient expression. The efficiency of infection was analyzed with flow cytometry using a BD FACSCanto system.

Growth of cells in Matrigel. Growth factor reduced Matrigel basement membrane matrix (BDBiosciences, USA) was diluted to a concentration of $6.0 \mathrm{mg} / \mathrm{ml}$ with serum-free medium (DMEM) and stored at $-80^{\circ} \mathrm{C}$. Before use, diluted Matrigel was thawed on ice overnight. $200 \mu \mathrm{l}$ of Matrigel solution was added into each well of a 24well plate and incubated at $37^{\circ} \mathrm{C}$ for $30 \mathrm{~min}$ to solidify. A single cell suspension $(5,000$ cells $/ 10 \mu \mathrm{l})$ was then mixed with $190 \mu \mathrm{l}$ of Matrigel at $4^{\circ} \mathrm{C}$ and added to the solidified bottom layer. The plates were incubated at $37^{\circ} \mathrm{C}$ for $30 \mathrm{~min}$, and then $500 \mu \mathrm{l}$ of complete medium was added on top ${ }^{40}$. Growth of cells in Matrigel was then observed during the following days. Triplicates were performed for each group.

Soft agar colony formation assay. Soft agar colony formation assay was performed in $35 \mathrm{~mm}$ dishes. Four $\mathrm{ml}$ of $0.6 \%$ autoclaved noble agar (Difco, USA) were added into each dish and left to solidify. Then $1.5 \mathrm{ml}$ of $0.3 \%$ agar $\left(40^{\circ} \mathrm{C}\right)$, containing 30,000 cells, were dispensed evenly on top of the solidified agar. One $\mathrm{ml}$ of full medium was added carefully to each dish after the agar had solidified. Fresh medium was supplemented twice a week. Random pictures ( 5 fields for each dish) were taken after 14 days, using an AxioCam MRm camera attached to an Axiovert 200M microscope (Carl Zeiss AG, Feldbach, Switzerland). Triplicates were performed for each group.

Transwell migration and invasion assays. The bottom side of 24-well polycarbonate membrane transwell inserts ( $8 \mu \mathrm{m}$ pore size; Corning Life Science, USA) was coated with $50 \mu \mathrm{l}$ of fibronectin $(10 \mu \mathrm{g} / \mathrm{ml})$ and incubated for 1 hour at room temperature, then blocked with $50 \mu \mathrm{l}$ of BSA $(100 \mu \mathrm{g} / \mathrm{ml})$ for 1 hour. For invasion assays, the upper side of the inserts was further coated with $50 \mu \mathrm{l}$ of Matrigel $(1 \mathrm{mg} / \mathrm{ml}$, diluted in serum-free DMEM). Cells ( $1 \times 10^{6}$ cells $\left./ \mathrm{ml} ; 100 \mu \mathrm{l}\right)$ were seeded in serum-free DMEM medium containing $0.2 \%$ BSA into the upper chambers, and $500 \mu \mathrm{l}$ of DMEM medium supplemented with $10 \%$ FBS was carefully added into the lower chambers as a chemo-attractant. After the indicated time, cells on the upper side of the insert were removed with cotton swabs, and those on the underside were stained with Hoechst 33342 (Sigma) and photographed using an AxioCam MRm camera attached to an Axiovert 200M microscope. Random pictures (5 fields per insert) were taken, and the cells on the underside were counted. Triplicates were performed for each group.

ShRNA-mediated RNA interference. MISSION lentiviral shRNA constructs, including the non-targeting shRNA control vector (SHC002), shULK1 (TRCN0000028755) and shAtg7 (TRCN0000092163) MISSION shRNA, were obtained from Sigma. After infection with shRNA lentivirus, cells were selected with 1 $\mu \mathrm{g} / \mathrm{ml}$ of puromycin (Sigma) for one week, and thereafter maintained in $0.5 \mu \mathrm{g} / \mathrm{ml}$ of puromycin.

Glucose starvation assay. Cells (30,000 cells per well) were seeded into 24 -well tissue culture plates in triplicates 1 day prior to starvation. For the starvation assay, growth medium was aspirated, and cells were carefully washed twice with phosphate buffered saline (PBS; Invitrogen) containing $\mathrm{Ca}^{2+}$ and $\mathrm{Mg}^{2+} .500 \mu$ l of glucose-deficient DMEM medium (Gibco), supplemented with 10\% FBS, was added into each well. After the indicated time, Hoechst 33342, a blue-fluorescent dye that stains all cells and propidium iodide (PI; Sigma), a red-fluorescent dye that stains dead cells, were added to culture media at a final concentration of $2 \mu \mathrm{g} / \mathrm{ml}$. After incubation for 5 minutes, 3 fields were randomly selected, and one picture in the blue channel and one in the red channel were acquired for each field using an AxioCam MRm camera attached to an Axiovert 200M microscope. Cells in each field were counted using the ImageJ software (http:// http://rsbweb.nih.gov/ij/), and the percentage of dead cells was calculated. ImageJ was also used to adjust image brightness, and to overlay blue and red channels.

Transfection and dual-luciferase reporter assays. One day prior to transfection, B16F1 cells were seeded in triplicates into 24-well plates at 30,000 cells per well. For transfection, $75 \mathrm{ng}$ psiCHECK-3'UTR plus $425 \mathrm{ng}$ miRNA plasmid were complexed with $1.5 \mu \mathrm{g}$ polyethylenimine for $20 \mathrm{mins}$ at room temperature in $50 \mu \mathrm{l}$ of growth medium without serum or antibiotics, and added drop-wise into each well. Thirty-six hours post transfection, a dual-luciferase assay was carried out according to the manufacturer's protocol (Promega). Briefly, cells were lysed in 1x Passive Lysis Buffer ( $100 \mu \mathrm{l}$ for each well) with shaking for $20 \mathrm{~min}$ at room temperature. Then $5 \mu \mathrm{l}$ of lysate was transferred into a well in a white 96-well assay plate, with 2 technical replicates. The Firefly and Renilla luciferase activity was measured with a DualLuciferase Reporter Assay (Promega), using an LMax384-II luminometer (Molecular Devices) equipped with two-channel automatic injections.

Western blot analyses. For preparation of cell lysates for Western blots, cells were cultured in $60-\mathrm{mm}$ dishes, $1 \times 10^{6}$ cells per dish, and were glucose starved in the presence or absence of E64d (Sigma, $10 \mu \mathrm{g} / \mathrm{ml}$ ) and pepstatin A (Sigma, $10 \mu \mathrm{g} / \mathrm{ml}$ ), or were treated with rapamycin (Sigma, $0.5 \mu \mathrm{g} / \mathrm{ml}$ ) for 24 hours. Cells were collected using cell scrapers on ice and were lysed. Cell lysates were boiled in Laemmli buffer and loaded onto NuPAGE Bis-Tris 4-12\% gradient precast gels (Invitrogen). Blots were incubated with the respective primary antibodies diluted in PBS containing 1\% BSA overnight at $4{ }^{\circ} \mathrm{C}$ and were then washed and incubated with the appropriate secondary antibodies and developed using the Amersham ECL Plus kit (GE Healthcare). Anti-Actb (Abcam, ab8227), anti-ULK1 (Sigma, A7481), anti-Atg5 (Sigma, A0731), anti-Atg7 (Sigma, A2856), anti-Atg16L1 (Sigma, A7356), anti-Becn (Cell signaling, 3738), anti-LC3 (Novus Biologicals, NB100-2220) and horseradish peroxidase-linked donkey anti-rabbit IgG (GE healthcare, NA9340V) were used. Bands were quantified using ImageJ after calibration.

Statistical analyses. Statistical analyses were performed using Prism version 5.0c for Mac (GraphPad Software, Inc.). Data are shown as means \pm SD and were analyzed with a two-tailed unpaired Student's $t$-test. Differences were considered statistically significant when $\mathrm{p}<0.05$

1. Garzon, R., Calin, G. A. \& Croce, C. M. MicroRNAs in cancer. Annu Rev Med 60, 167-179 (2009)

2. Garzon, R., Marcucci, G. \& Croce, C. M. Targeting microRNAs in cancer: rationale, strategies and challenges. Nat Rev Drug Discov 9, 775-789 (2010).

3. Levine, B. \& Kroemer, G. Autophagy in the pathogenesis of disease. Cell 132, 27-42 (2008)

4. Klionsky, D. J. Cell biology: regulated self-cannibalism. Nature 431, 31-32 (2004).

5. Liang, C. \& Jung, J. U. Autophagy genes as tumor suppressors. Curr Opin Cell Biol 22, 226-233 (2010)

6. Aita, V. M. et al. Cloning and genomic organization of beclin 1 , a candidate tumor suppressor gene on chromosome 17q21. Genomics 59, 59-65 (1999).

7. Qu, X. et al. Promotion of tumorigenesis by heterozygous disruption of the beclin 1 autophagy gene. J Clin Invest 112, 1809-1820 (2003).

8. Yue, Z., Jin, S., Yang, C., Levine, A. J. \& Heintz, N. Beclin 1, an autophagy gene essential for early embryonic development, is a haploinsufficient tumor suppressor. Proc Natl Acad Sci U S A 100, 15077-15082 (2003).

9. Liersch, R. et al. Analysis of a novel highly metastatic melanoma cell line identifies osteopontin as a new lymphangiogenic factor. Int J Oncol 41, 1455-1463 (2012).

10. Rittling, S. R. \& Chambers, A. F. Role of osteopontin in tumour progression. Br J Cancer 90, 1877-1881 (2004).

11. Volinia, S. et al. A microRNA expression signature of human solid tumors defines cancer gene targets. Proc Natl Acad Sci U S A 103, 2257-2261 (2006).

12. Suh, M. R. et al. Human embryonic stem cells express a unique set of microRNAs. Dev Biol 270, 488-498 (2004).

13. Sakahira, H., Enari, M. \& Nagata, S. Cleavage of CAD inhibitor in CAD activation and DNA degradation during apoptosis. Nature 391, 96-99 (1998).

14. Taylor, R. C., Cullen, S. P. \& Martin, S. J. Apoptosis: controlled demolition at the cellular level. Nat Rev Mol Cell Bio 9, 231-241 (2008).

15. Chen, A. Y., Yu, C., Bodley, A., Peng, L. F. \& Liu, L. F. A new mammalian DNA topoisomerase I poison Hoechst 33342: cytotoxicity and drug resistance in human cell cultures. Cancer Res 53, 1332-1337 (1993).

16. Aki, T., Yamaguchi, K., Fujimiya, T. \& Mizukami, Y. Phosphoinositide 3-kinase accelerates autophagic cell death during glucose deprivation in the rat cardiomyocyte-derived cell line H9c2. Oncogene 22, 8529-8535 (2003).

17. Hardie, D. G. AMPK and autophagy get connected. EMBO J 30, 634-635 (2011).

18. Kabeya, Y. et al. LC3, a mammalian homologue of yeast Apg8p, is localized in autophagosome membranes after processing. Embo Journal 19, 5720-5728 (2000).

19. Mizushima, N., Yoshimori, T. \& Levine, B. Methods in mammalian autophagy research. Cell 140, 313-326 (2010).

20. Seglen, P. O. \& Gordon, P. B. 3-Methyladenine: specific inhibitor of autophagic/ lysosomal protein degradation in isolated rat hepatocytes. Proc Natl Acad Sci US A 79, 1889-1892 (1982). 
21. Blommaart, E. F., Krause, U., Schellens, J. P., Vreeling-Sindelarova, H. \& Meijer, A. J. The phosphatidylinositol 3-kinase inhibitors wortmannin and LY294002 inhibit autophagy in isolated rat hepatocytes. Eur J Biochem 243, 240-246 (1997).

22. Yang, C. H., Yue, J. M., Pfeffer, S. R., Handorf, C. R. \& Pfeffer, L. M. MicroRNA miR-21 regulates the metastatic behavior of B16 melanoma cells. J Biol Chem 286, 39172-39178 (2011)

23. Levy, C. et al. Intronic miR-211 assumes the tumor suppressive function of its host gene in melanoma. Mol Cell 40, 841-849 (2010).

24. Lewis, B. P., Burge, C. B. \& Bartel, D. P. Conserved seed pairing, often flanked by adenosines, indicates that thousands of human genes are microRNA targets. Cell 120, 15-20 (2005)

25. Nielsen, C. B. et al. Determinants of targeting by endogenous and exogenous microRNAs and siRNAs. RNA 13, 1894-1910 (2007).

26. Klionsky, D. J. \& Emr, S. D. Cell biology - Autophagy as a regulated pathway of cellular degradation. Science 290, 1717-1721 (2000).

27. Yang, Z. S. et al. Rapamycin inhibits lung metastasis of B16 melanoma cells through down-regulating alphav integrin expression and up-regulating apoptosis signaling. Cancer Sci 101, 494-500 (2010).

28. Wang, Y. et al. Embryonic stem cell-specific microRNAs regulate the G1-S transition and promote rapid proliferation. Nat Genet 40, 1478-1483 (2008).

29. Zheng, G. X. et al. A latent pro-survival function for the mir-290-295 cluster in mouse embryonic stem cells. PLoS Genet 7, e1002054 (2011).

30. Palmer, R. D. et al. Malignant germ cell tumors display common microRNA profiles resulting in global changes in expression of messenger RNA targets. Cancer Res 70, 2911-2923 (2010).

31. Voorhoeve, P. M. et al. A genetic screen implicates miRNA-372 and miRNA-373 as oncogenes in testicular germ cell tumors. Cell 124, 1169-1181 (2006).

32. Sato, K. et al. Autophagy is activated in colorectal cancer cells and contributes to the tolerance to nutrient deprivation. Cancer Res 67, 9677-9684 (2007).

33. Yu, L. et al. Regulation of an ATG7-beclin 1 program of autophagic cell death by caspase-8. Science 304, 1500-1502 (2004)

34. Fidler, I. J. et al. Survival of cancer cells is maintained by EGFR independent of its kinase activity. Cancer Cell 13, 385-393 (2008).

35. Fung, C., Lock, R., Gao, S., Salas, E. \& Debnath, J. Induction of autophagy during extracellular matrix detachment promotes cell survival. Mol Biol Cell 19, 797-806 (2008)
36. Folkman, J. Angiogenesis. Annu Rev Med 57, 1-18 (2006).

37. White, E. Role of the metabolic stress responses of apoptosis and autophagy in tumor suppression. Ernst Schering Found Symp Proc 4, 23-34 (2007).

38. Hanahan, D. \& Weinberg, R. A. Hallmarks of cancer: the next generation. Cell 144, 646-674 (2011).

39. Reich, M. et al. GenePattern 2.0. Nat Genet 38, 500-501 (2006).

40. Wu, M. et al. Repression of BCL2 by the tumor suppressor activity of the lysyl oxidase propeptide inhibits transformed phenotype of lung and pancreatic cancer cells. Cancer Res 67, 6278-6285 (2007).

\section{Acknowledgements}

We thank Dr. Jonathan Hall for helpful discussions. This work was supported by Swiss National Science Foundation grants 3100A0-108207 and 31003A_130627, Commission of the European Communities grant LSHC-CT-2005-518178, Advanced European Research Council Grant LYVICAM, Krebsliga Schweiz and Krebsliga Zurich (to M.D.).

\section{Author contributions}

Y.C. and M.D. designed the experiments and wrote the manuscript. Y.C. performed the experiments and analyzed the data. R.L. generated the melanoma cell lines used in the study. All authors reviewed the manuscript.

\section{Additional information}

Supplementary information accompanies this paper at http://www.nature.com/ scientificreports

Competing financial interests: The authors declare no competing financial interests.

License: This work is licensed under a Creative Commons

Attribution-NonCommercial-No Derivative Works 3.0 Unported License. To view a copy of this license, visit http://creativecommons.org/licenses/by-nc-nd/3.0/

How to cite this article: Chen, Y., Liersch, R. \& Detmar, M. The miR-290-295 cluster suppresses autophagic cell death of melanoma cells. Sci. Rep. 2, 808; DOI:10.1038/ srep00808 (2012). 